\title{
Differential approximation results for the traveling salesman problem with distances 1 and 2
}

\author{
Jérôme Monnot Vangelis Th. Paschos* Sophie Toulouse \\ \{monnot, paschos, toulouse\}@lamsade.dauphine.fr
}

\begin{abstract}
We prove that both minimum and maximum traveling salesman problems on complete graphs with edge-distances 1 and 2 (denoted by min_TSP12 and max_TSP12, respectively) are approximable within 3/4. Based upon this result, we improve the standard approximation ratio known for maximum traveling salesman with distances 1 and 2 from $3 / 4$ to $7 / 8$. Finally, we prove that, for any $\epsilon>0$, it is NP-hard to approximate both problems better than within $741 / 742+\epsilon$. The same results hold when dealing with a generalization of min and max_TSP12, where instead of 1 and 2, edges are valued by $a$ and $b$.
\end{abstract}

\section{Introduction}

Given a complete graph on $n$ vertices, denoted by $K_{n}$, with edge distances either 1 or 2 the minimum traveling salesman problem (min_TSP12) consists of minimizing the cost of a Hamiltonian cycle, the cost of such a cycle being the sum of the distances on its edges (in other words, in finding a Hamiltonian cycle containing a maximum number of 1-edges). The maximum traveling salesman problem (max_TSP) consists of maximizing the cost of a Hamiltonian cycle (in other words, of finding a Hamiltonian cycle containing a maximum number of 2-edges). A generalization of TSP12, denoted by TSPab, is the one where the edge-distances are either $a$, or $b, a<b$. Both min_ and max_TSP12, and TSP $a b$ are NP-hard.

Given an instance $I$ of an NP optimization (NPO) problem $\Pi$ and a polynomial time approximation algorithm A feasibly solving $\Pi$, we will denote by $\omega(I), \lambda_{\mathbf{A}}(I)$ and $\beta(I)$ the values of the worst solution of $I$, of the approximated one (provided by A when running on $I$ ), and the optimal one for $I$, respectively. Generally (see [8]), the quality of an approximation algorithm for an NPhard minimization (resp., maximization) problem $\Pi$ is expressed by the ratio (called standard in what follows) $\rho_{\mathrm{A}}(I)=\lambda(I) / \beta(I)$, and the quantity $\rho_{\mathrm{A}}=\inf \left\{r: \rho_{\mathrm{A}}(I)<r, I\right.$ instance of $\left.\Pi\right\}$ (resp., $\rho_{\mathrm{A}}=\sup \left\{r: \rho_{\mathrm{A}}(I)>r, I\right.$ instance of $\left.\left.\Pi\right\}\right)$ constitutes the approximation ratio of $\mathrm{A}$ for $\Pi$. Another approximation-quality criterion used by many researchers $([2,1,3,4,13,14])$ is what in $[6,5]$ we call differential-approximation ratio. It measures how the value of an approximate solution is placed in the interval between $\omega(I)$ and $\beta(I)$. More formally, the differentialapproximation ratio of an algorithm A is defined as $\delta_{\mathrm{A}}(I)=|\omega(I)-\lambda(I)| /|\omega(I)-\beta(I)|$. The quantity $\delta_{\mathrm{A}}=\sup \left\{r: \delta_{\mathrm{A}}(I)>r, I\right.$ instance of $\left.\Pi\right\}$ is the differential approximation ratio of A for $\Pi$. In [2], the term "trivial solution" is used to denote the solution realizing the worst among the feasible solution-values of an instance. Moreover, all the examples in [2] carry over NP-hard problems for which worst solution can be trivially computed. This is for example the case of maximum independent set where, given a graph, the worst solution is the empty set, or of minimum vertex cover, where the worst solution is the vertex-set of the input-graph, or even of the

\footnotetext{
${ }^{*}$ LAMSADE, Université Paris-Dauphine, Place du Maréchal De Lattre de Tassigny, 75775 Paris Cedex 16, France
} 
minimum graph-coloring where one can trivially color the vertices of the input-graph using a distinct color per vertex. On the contrary, for TSP things are very different. Let us take for example min_TSP. Here, given a graph $K_{n}$, the worst solution for $K_{n}$ is a maximum total-distance Hamiltonian cycle, i.e., the optimal solution of max_TSP in $K_{n}$. The computation of such a solution is very far from being trivial since max_TSP is NP-hard. Obviously, the same holds when one considers max_TSP and tries to compute a worst solution for its instance, as well as for optimum satisfiability, for minimum maximal independent set and for many other well-known NP-hard problems. In order to remove ambiguities about the concept of the worst-value solution of an instance $I$ of an NPO problem $\Pi$, we will define it as the optimal solution opt $\left(\Pi^{\prime}\right)$ of an NPO problem $\Pi^{\prime}$ having the same set of instances and feasibility constraints as $\Pi$ verifying

$$
\operatorname{opt}\left(\Pi^{\prime}\right)= \begin{cases}\max & \operatorname{opt}(\Pi)=\min \\ \min & \operatorname{opt}(\Pi)=\max \end{cases}
$$

In general, no apparent links exist between standard and differential approximations in the case of minimization problems, in the sense that there is no evident transfer of a positive, or negative, result from one framework to the other. Hence, a "good" differential-approximation result implies nothing for the behavior of the approximation algorithm studied when dealing with the standard framework and vice versa. When dealing with maximization problems, we show in [10] that the approximation of a maximization NPO problem $\Pi$ within differential-approximation ratio $\delta$ implies its approximation within standard-approximation ratio $\delta$.

The best known standard-approximation ratio known for min_TSP12 is $7 / 6([11])$, while the best known standard inapproximability bound is $743 / 742-\epsilon$, for any $\epsilon>0$ ([7]). On the other hand, the best known standard-ratio max_TSP is 3/4 ([12]). To our knowledge, no better result is known in standard approximation for max_TSP12. Furthermore, no special study of TSPab has been performed until now (a trivial standard-approximation ratio of $b / a$ or $a / b$ is in any case very easily deduced for min_or max_TSP $a b$ ).

Here we show that min_ and max_TSP12, and min_ and max_TSP $a b$ are all equi-approximable within $3 / 4$ for the differential approximation. We also prove that all these problems cannot be approximated better than within $741 / 742+\epsilon$, for any $\epsilon>0$. By the equi-approximability of min_TSP12, max_TSP12, min_TSP $a b$ and $\max _{-}$TSP $a b$, the results obtained for the case of min_TSP12 apply to the rest of the problems above. Finally, we improve the standardapproximation ratio of max_TSP12 from $3 / 4([12])$ to $7 / 8$.

In what follows, we will denote by $V=\left\{v_{1}, \ldots, v_{n}\right\}$ the vertex-set of $K_{n}$, by $E$ its edge-set, and for $v_{i} v_{j} \in E$, we denote by $d\left(v_{i}, v_{j}\right)$ the distance of the edge $v_{i} v_{j} \in E$; we consider that the distance-vector is symmetric and integer. Given a feasible TSP-solution $T\left(K_{n}\right)$ of $K_{n}$ (both min and max_TSP have the same set of feasible solutions), we denote by $d\left(T\left(K_{n}\right)\right)$ its (objective) value. Given a graph $G$, we denote by $V(G)$ its vertex-set. Finally, given any set $C$ of edges, we denote by $d(C)$ the total distance of $C$, i.e., the quantity $\sum_{v_{i} v_{j} \in C} d\left(v_{i}, v_{j}\right)$.

\section{Differential-approximation preserving reductions for TSP12}

In this section we give a differential-approximation preserving result that will be used later.

Theorem 1. min_TSP12, max_TSP12, min_TSPab and max_TSPab are all equi-approximable for the differential approximation.

Proof. In order to prove the theorem we will prove the following stronger lemma.

Lemma 1. Consider any instance $I=\left(K_{n}, \vec{d}\right)$ (where $\vec{d}$ denotes the edge-distance vector of $K_{n}$ ). Then, any legal transformation $\vec{d} \mapsto \gamma \cdot \vec{d}+\eta \cdot \overrightarrow{1}$ of $\vec{d}(\gamma, \eta \in \mathbb{Q})$ produces differentially equiapproximable TSP-problems. 
Proof of lemma 1. Suppose that TSP can be approximately solved within differentialapproximation ratio $\delta$ and remark that both the initial and the transformed instance have the same set of feasible solutions. By the transformation considered, the value $d\left(T\left(K_{n}\right)\right)$ of any feasible tour $T\left(K_{n}\right)$ is affinely transformed into $\gamma d\left(T\left(K_{n}\right)\right)+\eta n$. Since differential-approximation ratio is stable under affine transformation, the equi-approximability of the original and of the transformed problem is immediately deduced, concluding so the proof of lemma 1.

We are ready now to continue the proof of theorem 1. In order to prove that min_TSP12 and max_TSP12 are equi-approximable, it suffices to apply lemma 1 proved just above with $\gamma=-1$ and $\eta=3$. On the other hand, in order to prove that min_ or max_TSP12 reduces to min_or max_TSP $a b$, we apply lemma 1 with $\gamma=1 /(b-a)$ and $\eta=(b-2 a) /(b-a)$, while for the converse reduction we apply lemma 1 with $\gamma=b-a$ and $\eta=2 a-b$. Since the reductions presented are transitive and composable, the equi-approximability of the pairs (min_TSP12, max_TSP12) and (TSP12, TSPab) proves the theorem.

For reasons of simplicity, we deal, in what follows, with min_TSP12. The differentialapproximation results obtained can be immediately transferred, by theorem 1, to max_TSP12, min_TSP $a b$ and max_TSPab.

\section{Approximating min_TSP12}

Let us first recall that, given a graph $G$, a 2-matching is a set $M$ of edges of $G$ such that if $V(M)$ is the set of the endpoints of $M$, the vertices of the graph $(V(M), M)$ have degree at most 2; in other words, the graph $(V(M), M)$ is a collection of cycles and simple paths. A 2-matching is optimal if it is the largest over all the 2-matchings of $G$. It is called perfect if any vertex of the graph $(V(M), M)$ has degree equal to 2, i.e., if it constitutes a partition of $V(M)$ into cycles in $G$. Remark that determining a maximum 2-matching in a graph $G$ is equivalent to determining a minimum total-distance vertex-partition into cycles into $G \cup \bar{G}$ (the complement of $G$ ), where the edges of $G$ are considered of distance 1 and the ones of $\bar{G}$ of distance 2 .

As shown in [9], an optimal triangle-free 2-matching can be computed in polynomial time. As mentioned above, this amounts to computing a triangle-free minimum-distance collection of cycles in a complete graph $K_{n}$ with edge-distances 1 and 2 . Let us denote by $M$ such a collection. Starting from $M$, we will progressively patch its cycles in order to finally obtain a unique Hamiltonian cycle in $K_{n}$.

\subsection{Preprocessing $M$}

We first define two operations, namely the 2-exchange and the 2-patching, implying two vertexdisjoint cycles of a 2-matching.

Definition 1. Let $C_{1}$ and $C_{2}$ be two vertex-disjoint cycles. Then:

- a 2-exchange is any replacement of two edges $v_{1} u_{1} \in C_{1}, v_{2} u_{2} \in C_{2}$ by the edges $v_{1} v_{2}$ and $u_{1} u_{2}$

- a 2-patching of $C_{1}$ and $C_{2}$ is any cycle $C$ resulting from a 2-exchange on $C_{1}$ and $C_{2}$, i.e., $C=\left(C_{1} \cup C_{2} \backslash\left\{v_{1} u_{1}, v_{2} u_{2}\right\}\right) \cup\left\{v_{1} v_{2}, u_{1} u_{2}\right\}$, for any pair $\left(v_{1} u_{1}, v_{2} u_{2}\right) \in C_{1} \times C_{2}$.

A 2-matching minimal with respect to the 2-exchange operation will be called 2-minimal. In particular, if all edges have the same cost, then a 2-minimal matching is a tour.

Definition 2. A 2-matching $M=\left(C_{1}, C_{2}, \ldots, C_{|M|}\right)$ is 2-minimal if it verifies, $\forall\left(C_{i}, C_{j}\right) \in$ $M \times M, C_{i} \neq C_{j}, \forall v_{1} u_{1} \in C_{i}, \forall v_{2} u_{2} \in C_{j}, d\left(v_{1}, v_{2}\right)+d\left(u_{1}, u_{2}\right)>d\left(u_{1} v_{1}\right)+d\left(u_{2} v_{2}\right)$. 
In other words, a 2-matching $M$ is 2-minimal if any 2-patching of its cycles produces a 2matching of total distance strictly greater than the one of $M$. Starting from a 2-matching $\hat{M}$ transformation of $\hat{M}$ into a 2-minimal one $M$ can be performed in polynomial time by the following procedure.

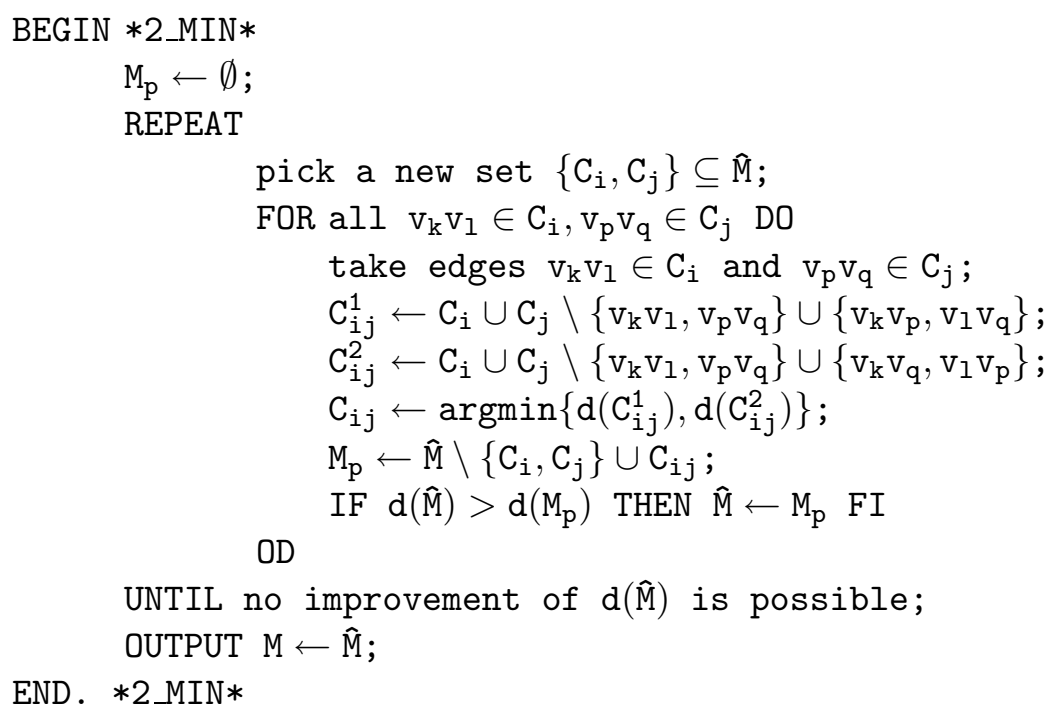

Moreover, suppose that there exist two distinct cycles $C$ and $C^{\prime}$ of $M$ (the output of the procedure 2_MIN), both containing 2-edges and denote by $u v \in C$ and $u^{\prime} v^{\prime} \in C^{\prime}$ two such edges. Then, $d\left(u u^{\prime}\right)+d\left(v v^{\prime}\right) \geqslant 4$, while $d(u v)+d\left(u^{\prime} v^{\prime}\right)=4$, a contradiction. So, the following proposition holds.

Proposition 1. In any 2-minimal 2-matching, at most one of its cycles contains 2-edges.

Remark 1. If the size of a 2-minimal triangle-free 2-matching $M$ is 1 , then, since a Hamiltonian tour is a special case of triangle-free 2-matching, $M$ is an optimal min_TSP12-solution. Hence, in what follows we will suppose 2-matchings of size at least 2 .

Assume now a 2-minimal triangle-free 2-matching $M=\left(C_{1}, \ldots, C_{p}, C_{0}\right)$, verifying remark 1 , where $C_{0}$ is the unique cycle of $M$ (if any) containing 2-edges. Construct a graph $H=\left(V_{H}, E_{H}\right)$; $V_{H}=\left\{w_{1}, \ldots, w_{p}\right\}$ contains a vertex per cycle of $M$ and, for $i \neq j, w_{i} w_{j} \in E_{H}$ iff $\exists(u, v) \in C_{i} \times C_{j}$ such that $d(u, v)=1$. Consider a maximum matching $M_{H},\left|M_{H}\right|=q$, of $H$. With any edge $w_{i^{s}} w_{j^{s}}$ of $M_{H}$ we associate the pair $\left(C_{i^{s}}, C_{j^{s}}\right)$ of the corresponding cycles of $M$. So, $M$ can be described (up to renaming its cycles) as

$$
M=\bigcup_{s=1}^{q}\left\{C_{1}^{s}, C_{2}^{s}\right\} \bigcup_{t=1}^{r=p-2 q}\left\{C_{t}\right\} \bigcup\left\{C_{0}\right\}
$$

where for $s=1, \ldots, q, \exists e^{s} \in V\left(C_{1}^{s}\right) \times V\left(C_{2}^{s}\right)$ such that $d\left(e^{s}\right)=1$.

Consider $M$ as expressed in (1), denote by $V_{s}$ the set of the four vertices of $C_{1}^{s}$ and $C_{2}^{s}$ adjacent to the endpoints of $e^{s}$, and construct the bipartite graph $B=\left(V_{B}^{1} \cup V_{B}^{2}, E_{B}\right)$ where $V_{B}^{1}=\left\{w_{1}, \ldots, w_{r}\right\}$ (i.e., we associate a vertex with a cycle $\left.C_{t}, t=1, \ldots, r\right), V_{B}^{2}=\left\{w^{1}, \ldots, w^{q}\right\}$ (i.e., we associate a vertex with a pair $\left.\left(C_{1}^{s}, C_{2}^{s}\right), s=1, \ldots q\right)$ and, $\forall(t, s), w_{t} w^{s} \in E_{B}$ iff $\exists u \in C_{t}$, $\exists v \in V_{s}$ such that $d(u, v)=1$. Compute a maximum matching $M_{B},\left|M_{B}\right|=q^{\prime}$ in $B$. With any edge $w_{t} w^{s} \in M_{B}$ we associate the triple $\left(C_{1}^{s}, C_{2}^{s}, C_{t}\right)$. So, $M$ can be described (up to renaming its cycles) as

$$
M=\bigcup_{s=1}^{q^{\prime}}\left\{C_{1}^{s}, C_{2}^{s}, C_{3}^{s}\right\} \bigcup_{s=q^{\prime}+1}^{q}\left\{C_{1}^{s}, C_{2}^{s}\right\} \bigcup_{t=1}^{r^{\prime}=r-q^{\prime}}\left\{C_{t}\right\} \bigcup\left\{C_{0}\right\}
$$


where for $s=1, \ldots, q^{\prime}, \exists f^{s} \in V_{s} \times V\left(C_{3}^{s}\right)$ such that $d\left(f^{s}\right)=1$. In what follows we will reason with respect to $M$ as it has been expressed in (2).

\subsection{Computation and evaluation of the approximate solution and a lower bound for the optimal tour}

In the sequel, call s.d.e.p. a set of vertex-disjoint elementary paths, denote by PREPROCESS the procedure that starting from a 2-minimal triangle-free 2-matching $M$ leads to (2) and consider the following algorithm.

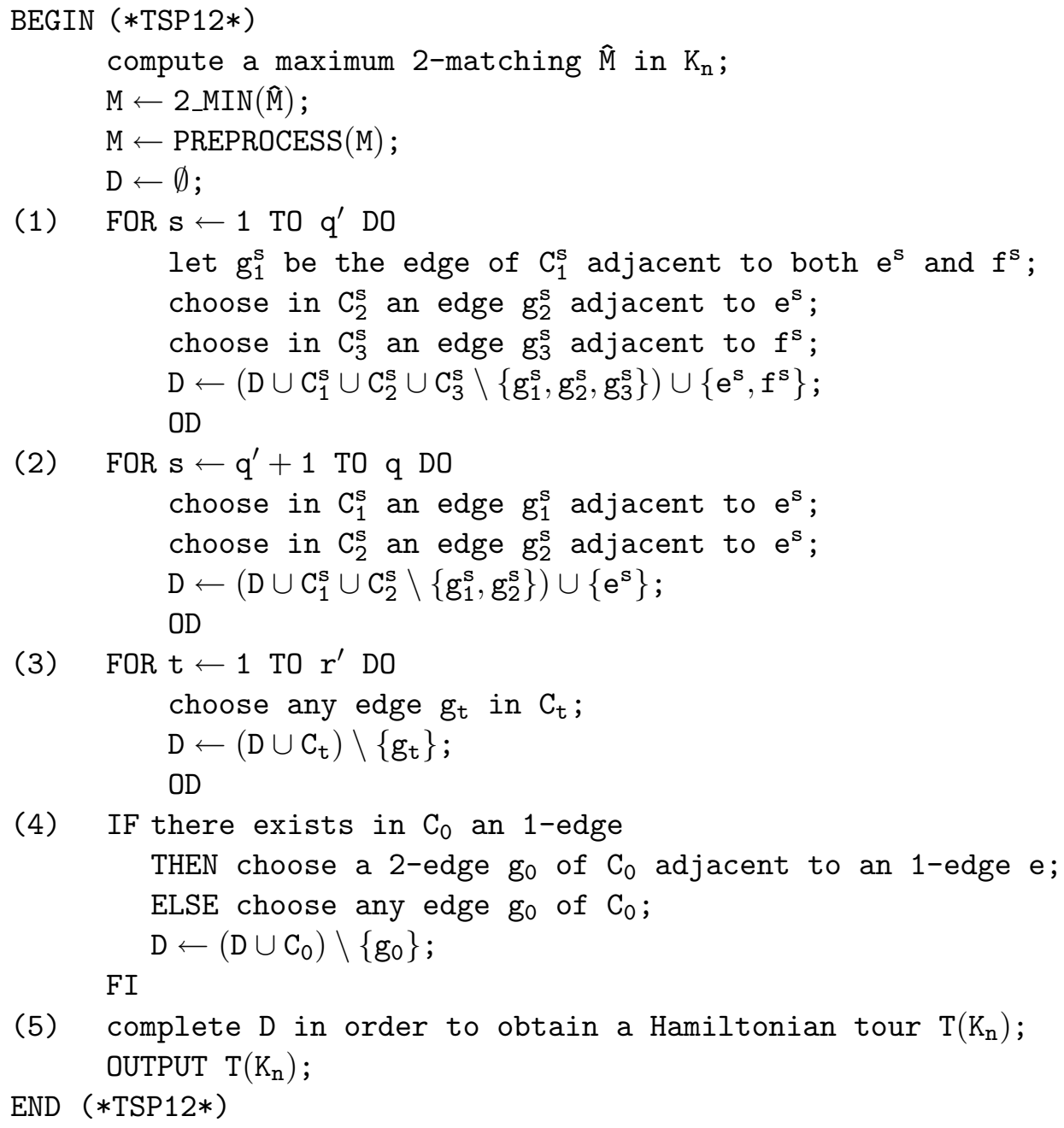

Clearly, both achievement of a 2-minimal triangle free 2-matching and PREPROCESS can be performed in polynomial time. Moreover, steps (1) to (4) are also executed in polynomial time. Finally, step (5) can be performed by arbitrarily ordering $(\bmod |D|)$ the chains of the s.d.e.p. $D$ and then, for $i=1, \ldots,|D|$, adding in $D$ the edge linking the "last" vertex of chain $i$ to the "first" vertex of chain $i+1$. Consequently, the whole algorithm TSP12 is polynomial. Finally, remark that $C_{0}$ may contain either only 2-edges, or both 1- and 2-edges. In the latter case, edge $g_{0}$ (in step 4) can be any 2-edge in $C_{0}$, adjacent to an 1-edge of $C_{0}$.

Lemma 2. $d\left(T\left(K_{n}\right)\right) \leqslant d(M)+q+r^{\prime}$.

Proof. During steps (1) to (4) of algorithm TSP12, set $D$ remains a s.d.e.p. At the end of step (4), $D$ contains $M$ minus the $3 q^{\prime}+2\left(q-q^{\prime}\right)+r^{\prime}=q^{\prime}+2 q+r^{\prime}$ 1-edges of the set 
$\cup_{s=1}^{q^{\prime}}\left\{g_{1}^{s}, g_{2}^{s}, g_{3}^{s}\right\} \cup_{s=q^{\prime}+1}^{q}\left\{g_{1}^{s}, g_{2}^{s}\right\} \cup_{s=1}^{r^{\prime}}\left\{g_{t}\right\}$ minus (if $C_{0} \neq \emptyset$ ) one 2-edge of $C_{0}$ plus the $2 q^{\prime}+\left(q-q^{\prime}\right)=$ $q^{\prime}+q$ 1-edges of the set $\cup_{s=1}^{q^{\prime}}\left\{e_{1}^{s}, f_{2}^{s}\right\} \cup_{s=q^{\prime}+1}^{q}\left\{e^{s}\right\}$. So $D$ is a s.d.e.p. of size $n-\left(q+r^{\prime}\right)-\mathbf{1}_{C_{0} \neq \emptyset}$ and of total distance $d(M)-\left(q+r^{\prime}\right)-2 . \mathbf{1}_{C_{0} \neq \emptyset}$. Completion of $D$ in order to obtain a tour in $K_{n}$, can be done by adding $q+r^{\prime}+\mathbf{1}_{C_{0} \neq \emptyset}$ new edges. Each of these new edges can be, at worst, of distance 2. We so have $d\left(T\left(K_{n}\right)\right) \leqslant d(M)-\left(q+r^{\prime}+2 . \mathbf{1}_{C_{0} \neq \emptyset}\right)+2\left(q+r^{\prime}+\mathbf{1}_{C_{0} \neq \emptyset}\right)=d(M)+q+r^{\prime}$, q.e.d. I

On the other hand, the optimal tour being a special triangle-free 2-matching, the following lemma holds immediately.

Lemma 3. $\beta\left(K_{n}\right) \geqslant d(M)$.

\subsection{Evaluation of the worst-value solution}

In what follows in this section we will exhibit a s.d.e.p., with all edges of distance 2 (called 2s.d.e.p.). Given such a s.d.e.p. $W$, one can proceed as in step (5) of algorithm TSP12 (section 3.2), in order to construct a Hamiltonian tour $T_{w}$ whose total distance is a lower bound for $\omega\left(K_{n}\right)$.

Denote by $E 2$ the set of 2-edges of cycle $C_{0}$. If $q=0$, i.e., $M_{H}=\emptyset$, and if $C_{0}=E 2$, then the tour computed by TSP12 is optimal.

Lemma 4. If $q=0$ and $C_{0}=E 2$, then $\delta_{\mathrm{TSP} 12}\left(K_{n}\right)=1$.

Proof. Let $k=\left|V\left(C_{0}\right)\right|=d(M)-n$ and set $V\left(C_{0}\right)=\left\{a_{1}, \ldots, a_{k}\right\}$. By the fact that $M$ is 2-minimal, all the edges of $K_{n}$ incident to these vertices have distance 2. On the other hand, between two distinct cycles in the set $\left\{C_{1}, \ldots, C_{p=r^{\prime}}\right\}$ of $M$, there exist only edges of distance 2 . Consider the family $\mathcal{F}=\left\{\left\{a_{1}\right\}, \ldots,\left\{a_{k}\right\}, V\left(C_{1}\right), \ldots V\left(C_{p}\right)\right\}$. By the above remarks, any edge linking vertices of two distinct sets of $\mathcal{F}$ is a 2-edge. Any feasible tour of $K_{n}$ (a posteriori an optimal one) integrates the $k+p$ sets of $\mathcal{F}$ by using at least $k+p$ 2-edges pairwise linking these sets. Hence, any tour uses at least $k+p$ 2-edges, so does tour $T\left(K_{n}\right)$ computed by algorithm TSP12, q.e.d.

So, we suppose in the sequel that $q=0 \Rightarrow C_{0} \neq E_{2}$. We will now prove the existence of a 2-s.d.e.p. $W$ of size $d(M)+4\left(q+r^{\prime}\right)-n$, where $M$ is as expressed by (2). In the sequel, a path with alternating vertices will denote a path such that no two consecutive vertices lie in the same cycle.

Proposition 2. Between two cycles $C_{a}$ and $C_{b}$ of $M$ of size at least $k$, there always exists a path with alternating vertices from $C_{a}$ and $C_{b}$, which contains at least $k$ 2-edges.

Proof. Let $\left\{a_{1}, \ldots, a_{k+1}\right\}$ and $\left\{b_{1}, \ldots, b_{k+1}\right\}$ be $k+1$ successive vertices of two distinct cycles $C_{a}$ and $C_{b}$ of size at least $k$ (possibly $a_{1}=a_{k+1}$ if $\left|V\left(C_{a}\right)\right|=k$ and $b_{1}=b_{k+1}$ if $\left|V\left(C_{b}\right)\right|=$ $k)$. We will show that there exists a path with alternating vertices from $C_{a}$ and $C_{b}$ of size $2 k-1$ and of distance at least $3 k-1$. Consider paths $C=\cup_{i=1}^{k}\left\{a_{i} b_{i}\right\} \cup_{i=1}^{k-1}\left\{a_{i+1} b_{i}\right\}$ and $D=\cup_{i=2}^{k+1}\left\{a_{i} b_{i}\right\} \cup_{i=1}^{k-1}\left\{a_{i} b_{i+1}\right\}$. By the 2-minimality of M we get:

$$
\begin{array}{llll}
\forall i=1, \ldots, k & \max \left\{d\left(a_{i}, b_{i}\right), d\left(a_{i+1}, b_{i+1}\right)\right\}=2 & \Rightarrow & d\left(a_{i}, b_{i}\right)+d\left(a_{i+1}, b_{i+1}\right) \geqslant 3 \\
\forall i=1, \ldots, k-1 & \max \left\{d\left(a_{i}, b_{i+1}\right), d\left(a_{i+1}, b_{i}\right)\right\}=2 \quad \Rightarrow \quad d\left(a_{i}, b_{i+1}\right)+d\left(a_{i+1}, b_{i}\right) \geqslant 3
\end{array}
$$

Summing the terms of the expression above member-by-member, one obtains:

$$
\begin{aligned}
& \sum_{i=1}^{k}\left(d\left(a_{i}, b_{i}\right)+d\left(a_{i+1}, b_{i+1}\right)\right)+\sum_{i=1}^{k-1}\left(d\left(a_{i+1}, b_{i}\right)+d\left(a_{i}, b_{i+1}\right)\right) \geqslant 6 k-3 \\
\Longleftrightarrow & d(C)+d(D) \geqslant 6 k-3 \Rightarrow \max \{d(C), d(D)\} \geqslant\left\lceil\frac{6 k-3}{2}\right\rceil=3 k-1
\end{aligned}
$$

Application of proposition 2 to any pair $\left(C_{1}^{s}, C_{2}^{s}\right)$ of $\mathrm{M}$ results in the following claim. 
Claim 1. $\forall s=1, \ldots, q$, there exists a 2-s.d.e.p. $W^{s}$ of size 4, alternating vertices of cycles $C_{1}^{s}$ and $C_{2}^{s}$, containing a vertex of $V_{s}$ whose degree with respect to $W^{s}$ is 1.

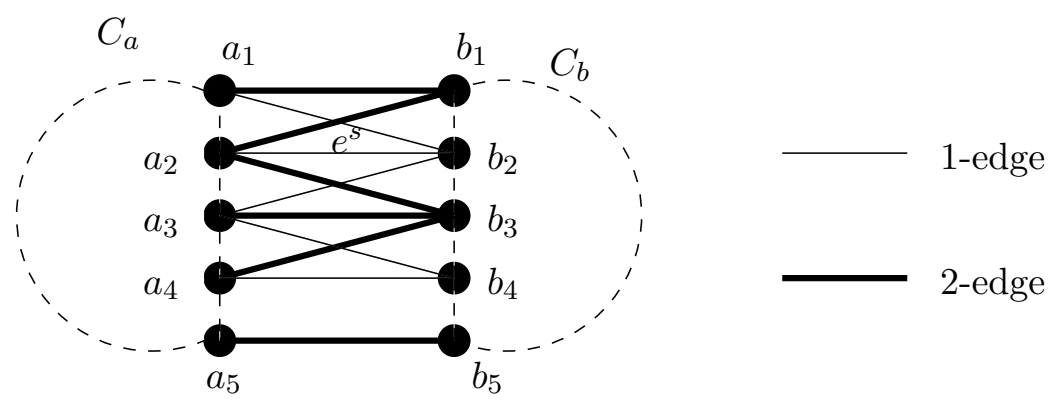

Figure 1: An example of claim 1.

In figure 1 , we show an application of claim 1 . We assume $e^{s}=a_{2} b_{2}$; then $\left\{a_{1}, b_{1}\right\} \subset V_{s}$. The 2-s.d.e.p. $W^{s}$ claimed is $\left\{b_{1} a_{2},\left(a_{3} b_{3}, b_{3} a_{4}\right), a_{5} b_{5}\right\}$ and the degree of $b_{1}$ with respect to $W^{s}$ is 1 .

Consider now the s.d.e.p. $W_{t}^{s}=W^{s} \cup W_{t}^{\prime s}$, where $W^{s}$ as in claim 1 and $W_{t}^{\prime s}$ is any path of size 4 with alternating vertices from $C_{3}^{s}$ and $C_{t}, s=1, \ldots, q^{\prime}, t=1, \ldots, r^{\prime}$. By the optimality of $M_{H}$, any edge linking vertices of $C_{3}^{s}$ to vertices of $C_{t}$ is a 2-edge. Consequently, $W_{t}^{s}$ is a 2-s.d.e.p. and the following claim holds.

Claim 2. $\forall s=1, \ldots, q^{\prime}, \forall t=1, \ldots, r^{\prime}$, there exists a 2-s.d.e.p. $W_{t}^{s}$ of size 8, alternating vertices of the cycles $C_{1}^{s}$ and $C_{2}^{s}$, and of the cycles $C_{3}^{s}$ and $C_{t}$.

For $s=q^{\prime}+1, \ldots, q, t=1, \ldots r^{\prime}$, consider the triplet $\left(C_{1}^{s}, C_{2}^{s}, C_{t}\right)$. Let $e^{s}=e_{1}^{s} e_{2}^{s}, V_{s}=$ $\left\{u_{1}^{s}, v_{1}^{s}, u_{2}^{s}, v_{2}^{s}\right\}$ and consider any four vertices $a_{t}, b_{t}, c_{t}$ and $d_{t}$ of $C_{t}$. By the optimality of $M_{B}$, any vertex of $C_{t}$ is linked to any vertex of $V^{s}$ exclusively by 2-edges. Moreover, the 2-minimality of $M$ implies that at least one of $u_{1}^{s} e_{2}^{s}$ and $e_{1}^{s} u_{2}^{s}$ is of distance 2. If we suppose $d\left(u_{1}^{s}, e_{2}^{s}\right)=2$ (figure 2), then the path $\left\{e_{2}^{s}, u_{1}^{s}, a_{t}, v_{1}^{s}, b_{t}, u_{2}^{s}, c_{t}, v_{2}^{s}, d_{t}\right\}$ is a 2-s.e.d.p. Hence, the following claim holds.

Claim 3. $\forall s=q^{\prime}+1, \ldots, q, \forall t=1, \ldots, r^{\prime}$, there exists a 2-s.d.e.p. $W_{t}^{s}$ of size 8, alternating vertices of the cycles $C_{1}^{s}, C_{2}^{s}$ and $C_{t}$.

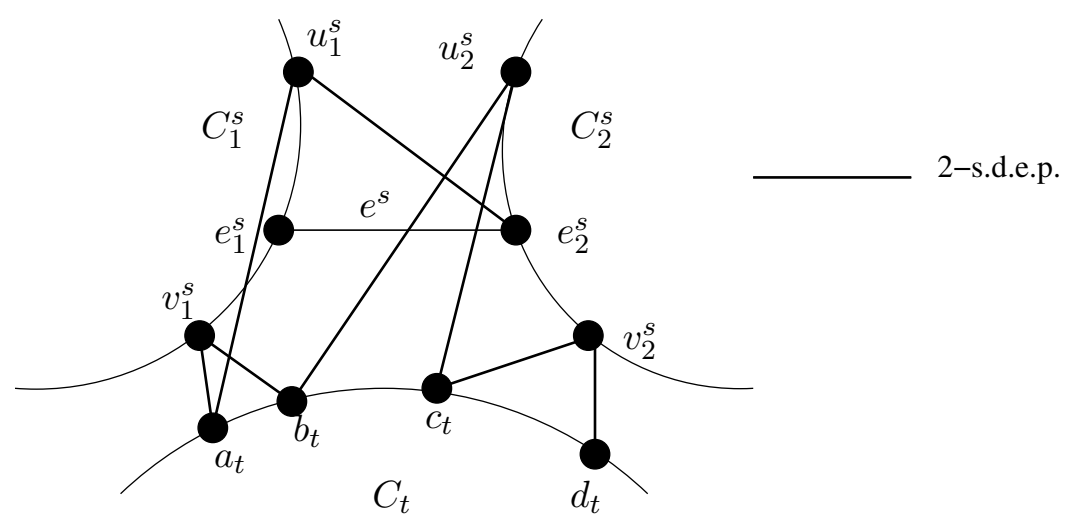

Figure 2: The 2-s.d.e.p. $W_{t}^{s}$ of claim 3 . 
Let $r^{\prime} \geqslant 2$ and consider, for $t=1, \ldots, r^{\prime}$, the (residual) cycles $C_{t}$. All edges between these cycles are of distance 2. If we denote by $a_{t}, b_{t}, c_{t}$ and $d_{t}$ four vertices of $C_{t}$, the path $\left\{a_{1}, \ldots, a_{t}, \ldots, a_{r^{\prime}}, b_{1}, \ldots, b_{t}, \ldots, b_{r^{\prime}}, c_{1}, \ldots, c_{t}, \ldots, c_{r^{\prime}}, d_{1}, \ldots, d_{t}, \ldots, d_{r^{\prime}}\right\}$ is a 2 -s.d.e.p. of size $4 r^{\prime}-1$ and the following claim holds.

Claim 4. If $r^{\prime} \geqslant 2$, then there exists a 2-s.d.e.p. $W^{r^{\prime}}$ of size $4 r^{\prime}-1$ alternating vertices of cycles $C_{t}, t=1, \ldots, r^{\prime}$.

Lemma 5. If $q \geqslant 1$, or if $\left[\left(C_{0} \neq E_{2}\right)\right.$ and $\left.(r \neq 1)\right]$, then $\delta_{\mathrm{TSP} 12}\left(K_{n}\right) \geqslant 3 / 4$.

Proof. Consider $M$ as expressed in (2). Let us denote by $W^{\prime}$ a 2-s.e.d.p. on $V \backslash V\left(C_{0}\right)$. From claims 1, 2, 3 and 4 :

$$
W^{\prime}=\left\{\begin{array}{lll}
\bigcup_{s=1}^{r^{\prime}} W_{s}^{s} \bigcup_{s=r^{\prime}+1}^{q} W^{s} & \left|W^{\prime}\right|=8 r^{\prime}+4\left(q-r^{\prime}\right)=4\left(q+r^{\prime}\right) & q \geqslant r^{\prime} \\
\bigcup_{s=1}^{q} W^{s} \bigcup W^{r^{\prime}} \bigcup\{\gamma\} & \left|W^{\prime}\right|=4 q+4 r^{\prime}-1+1=4\left(q+r^{\prime}\right) & r^{\prime}>q>0 \\
W^{r^{\prime}} & \left|W^{\prime}\right|=4 r^{\prime}-1=4\left(q+r^{\prime}\right)-1 & r^{\prime} \geqslant 2, q=0
\end{array}\right.
$$

In (3), $\gamma$ draws an edge linking a vertex of degree 1 with respect to $W^{r^{\prime}}$ to a vertex of degree 1 in $V\left(W^{\prime}\right)$. This last vertex can belong either to $V\left(C_{3}^{1}\right)$ if $q^{\prime} \geqslant 1$, or to $V_{q}$ otherwise. By the optimality of $M_{H}$ and $M_{B}, \gamma$ is a 2-edge. For the first line of (3), remark that if $q=r^{\prime}$, then $W^{\prime}$ is given by $\cup_{s=1}^{r^{\prime}} W_{s}^{s}$, and claims 2 and 3 conclude $W^{\prime}=8 r^{\prime}=4\left(r^{\prime}+q\right)$; otherwise $\left(q>r^{\prime}\right)$, claims 1, 2 and 3 conclude the first line of (3). For the second line $\left(r^{\prime}>q>0\right)$, since $r^{\prime}$ and $q$ are integers, we have $r^{\prime} \geqslant 2$. Then, by claim $4,\left|W^{r^{\prime}}\right| \geqslant 4 r^{\prime}-1$ and the expression of the second line follows from the fact that $\gamma$ is a 2-edge. For the third line of (3), claim 4 gives immediately the result. Hence, in any case, $W^{\prime}$ is a 2-s.d.e.p. verifying $\left|W^{\prime}\right|=4\left(q+r^{\prime}\right)-1$ if $q=0,\left|W^{\prime}\right|=4\left(q+r^{\prime}\right)$ otherwise.

We now construct a a 2-s.e.d.p. $W_{0}$ on $V\left(C_{0}\right)$. Let $g_{0}=u_{0} v_{0}$ be the edge removed from $C_{0}$ during the execution of step (4) of algorithm TSP12. If $C_{0} \neq E 2$, then $g_{0}$ has been chosen in such a way that one of its endpoints, say $v_{0}$, is adjacent in $C_{0}$ to an 1-edge. Let $\gamma^{\prime}$ be an edge linking $v_{0}$ to a vertex of degree 1 with respect to $W^{\prime}$ (such a vertex exists since $W^{\prime}$ is acyclic). By the 2-minimality of $M, d\left(\gamma^{\prime}\right)=2$. Set

$$
W_{0}=\left\{\begin{array}{lll}
E 2 \backslash\left\{g_{0}\right\} \cup\left\{\gamma^{\prime}\right\} & \left|W_{0}\right|=d(M)-n & C_{0}=E 2 \\
E 2 \cup\left\{\gamma^{\prime}\right\} & \left|W_{0}\right|=d(M)-n+1 & \text { otherwise }
\end{array}\right.
$$

Setting finally $W=W^{\prime} \cup W_{0}$, one obtains the 2-s.d.e.p. claimed at the beginning of section 3.3. We have $d(W)=4\left(q+r^{\prime}\right)-\mathbf{1}_{q=0}+d(M)-n+\mathbf{1}_{C_{0} \neq E 2} \geqslant d(M)-n+4\left(q+r^{\prime}\right)$, the last inequality holding because of the hypothesis $q=0 \Rightarrow C_{0} \neq E_{2}$ made just before lemma 2. Any completion of $W$ in a Hamiltonian cycle of $K_{n}$ (as in step (5) of algorithm TSP12) would produce a tour $T_{w}$ of total distance $d\left(T_{w}\right) \geqslant d(M)+4\left(q+r^{\prime}\right)$. So, $\omega\left(K_{n}\right) \geqslant d(M)+4\left(q+r^{\prime}\right)$, and combining this expression together with lemmata 2 and 3 , the differential ratio $3 / 4$ is immediately concluded.

Lemma 6. If $q=0$ and $r=1$ and $C_{0} \neq E_{2}$, then $\delta_{\mathrm{TSP} 12}\left(K_{n}\right) \geqslant 3 / 4$.

Proof. Recall that, by the optimality of $M_{H}$, if $q=0$ and $r=1$, all edges linking cycles $C_{0}$ and $C_{1}$ are of distance 2. Let $u_{0}, u_{1}$ and $u_{2}$ be three vertices of $C_{0}$ such that $d\left(u_{0}, u_{1}\right)=1$ and $d\left(u_{1}, u_{2}\right)=2$. We denote by $u_{2}$ a neighbor of $u_{1}$ with respect to $C_{0}$, and by $u_{3} \neq u_{1}$ the neighbor of $u_{2}$ in $C_{0}$. Let $a, b, c$ and $d$ be any four vertices of $C_{1}$, and let $W_{p}$ and $W_{m}$ be the sets $\left\{a u_{1}, u_{1} b, b u_{2}, u_{2} c\right\}$ and $\left\{u_{1} u_{2}\right\}$, respectively. 


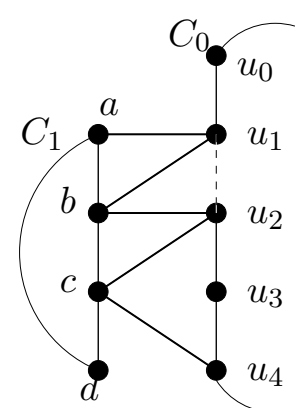

(a)

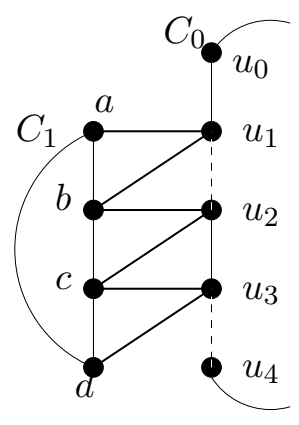

(b)

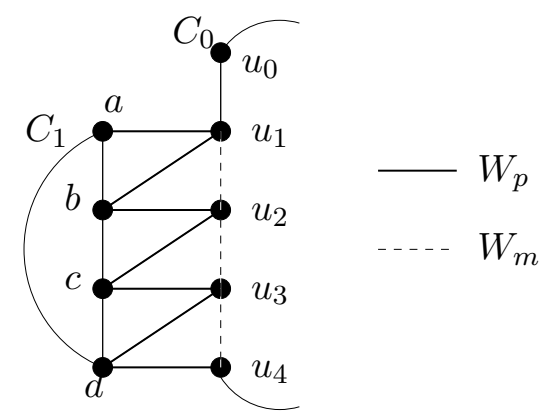

(c)

Figure 3:

If $u_{3}$ is adjacent to two 1 -edges in $C_{0}$, i.e., $d\left(u_{2}, u_{3}\right)=d\left(u_{3}, u_{4}\right)=1$, then set $W_{p}=W_{p} \cup\left\{c u_{4}\right\}$ (figure 3(a)).

If only one of $u_{2} u_{3}$ and $u_{3} u_{4}$ is of distance 2, then set $W_{p}=W_{p} \cup\left\{c u_{3}, u_{3} d\right\}$ and $W_{m}=$ $W_{m} \cup\left\{\operatorname{argmax}\left\{d\left(u_{2}, u_{3}\right), d\left(u_{3}, u_{4}\right)\right\}\right\}$. In figure $3(\mathrm{~b})$, sets $W_{p}$ and $W_{m}$ are shown for the case where $\operatorname{argmax}\left\{d\left(u_{2}, u_{3}\right), d\left(u_{3}, u_{4}\right)\right\}=u_{3} u_{4}$, i.e., $d\left(u_{3}, u_{4}\right)=2$ and $d\left(u_{2}, u_{3}\right)=1$.

Finally, if $u_{2} u_{3}$ and $u_{3} u_{4}$ are both of distance $2\left(d\left(u_{2}, u_{3}\right)=d\left(u_{3}, u_{4}\right)=2\right)$, then set $W_{p}=$ $W_{p} \cup\left\{c u_{3}, u_{3} d, d u_{4}\right\}$ and $W_{m}=W_{m} \cup\left\{u_{2} u_{3}, u_{3} u_{4}\right\}$ (figure $3(\mathrm{c})$ ).

In any of the above cases, we consider the 2-s.d.e.p. $W=E 2 \cup W_{p} \backslash W_{m}$ with $|W|=$ $d(M)-n+4=d(M)-n+4 r^{\prime}$. We then have $\omega\left(K_{n}\right) \geqslant d(M)+4 r^{\prime}$, and combining it with lemmata 2 and 3 , the differential ratio $3 / 4$ is concluded.

In all, combining lemmata $2,3,4$ and 6 , the following theorem can be immediately proved.

Theorem 2. min_TSP12 is approximable within differential-approximation ratio 3/4.

Theorems 1 and 2 induce the following corollary.

Corollary 1. min_TSP12, max_TSP12, min_TSPab and max_TSPab are approximable within differential-approximation ratio $3 / 4$.

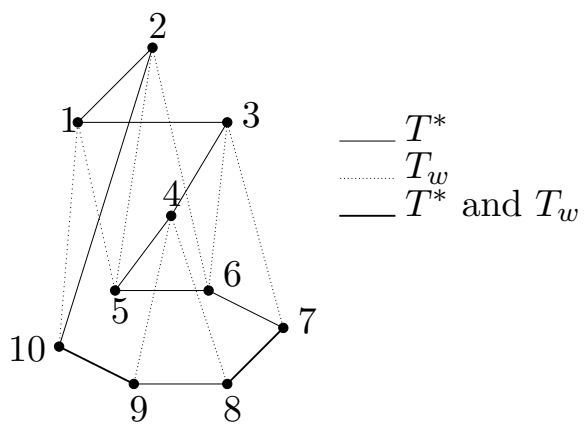

Figure 4: Tightness of the TSP12 approximation ratio.

Proposition 3. Ratio 3/4 is tight for TSP12

Proof. Consider two cliques and number their vertices by $\{1, \ldots, 4\}$ and by $\{5,6, \ldots, n+8\}$, respectively. Edges of both cliques have all distance 1 . Cross-edges $i j, i=1,3, j=5, \ldots, n+8$, 


\begin{tabular}{|c|c|c|c|}
\hline & $k$ & TSP12 & The algorithm of [11] \\
\hline 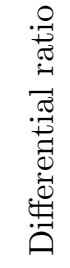 & $\begin{array}{l}3 \\
4 \\
5 \\
6\end{array}$ & $\begin{array}{c}0.931100364 \\
0.9000002 \\
0.920289696 \\
0.9222222\end{array}$ & $\begin{array}{c}0.846702091 \\
0.833333 \\
0.833333 \\
0.833333\end{array}$ \\
\hline 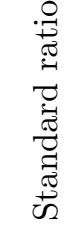 & $\begin{array}{l}3 \\
4 \\
5 \\
6\end{array}$ & $\begin{array}{c}0.923350955 \\
0.9094018 \\
0.92646313 \\
0.928178\end{array}$ & $\begin{array}{c}0.87013 \\
0.857143 \\
0.857143 \\
0.857143\end{array}$ \\
\hline
\end{tabular}

Table 1: A limited comparison between TSP12 and the algorithm of [11] on some worst-case instances of the latter.

are all of distance 2, while every other cross-edge is of distance 1. Unraveling of TSP12 will produce: $T=\{1,2,3,4,5,6, \ldots, n+7, n+8,1\}$ (cycle-patching on edges $(1,4)$ and $(5, n+8)$ ), while $T_{w}=\{1,5,2,6,3,7,4,8,9 \ldots, n+7, n+8,1\}$ (using 2-edges $(1,5),(6,3),(3,7)$ and $(n+8,1)$ ) and $T^{*}=\{1,2, n+8, n+7, \ldots, 5,4,3,1\}$ (using 1-edges $(4,5)$ and $(2, n+8)$ ). Consequently, $\delta_{\mathrm{TSP} 12}\left(K_{n+8}\right)=3 / 4$, q.e.d.

In figure 4 , the tours $T^{*}$ and $T_{w}$ of proposition 3 are shown for $n=2$. We assume $T=$ $\{1, \ldots, 10,1\}$.

Let us note that the differential approximation ratio of the $7 / 6$-algorithm of [11], when running on $K_{n+8}$, is also $3 / 4$. The authors of [11] exhibit a family of worst-case instances for their algorithm: one has $k$ cycles of length 4 arranged around a cycle of length $2 k$. We have performed a limited comparative study between their algorithm and ours, for $k=3,4,5,6$ (on 24 graphs). The average differential and standard approximation ratios for the two algorithms are presented in table 1.

Proposition 4. min_TSPab is approximable within standard-approximation ratio $\rho \leqslant(1+$ $((b-a) /(4 a))$. This ratio tends to $\infty$ when $a=o(b)$.

Proof. Revisit corollary 1. Differential ratio $3 / 4$ for min_TSPab implies $\lambda\left(K_{n}\right) / \beta\left(K_{n}\right) \leqslant$ $(3 / 4)+\left(\omega\left(K_{n}\right) /\left(4 \beta\left(K_{n}\right)\right)\right)$. Using $\omega\left(K_{n}\right) \leqslant b n$ and $\beta\left(K_{n}\right) \geqslant a n$, some easy algebra gives the result claimed.

Theorem 3. min_ and max_TSPab and min_ and max_TSP12 are inapproximable within differential-ratio of at least $742 / 743+\epsilon, \forall \epsilon>0$, unless $\boldsymbol{P}=\boldsymbol{N P}$.

Proof. Consider min_TSP12. Using $n \leqslant \beta\left(K_{n}\right) \leqslant \omega\left(K_{n}\right) \leqslant 2 n$, one can see that approximation of min_TSP12 within $\delta=1-\epsilon$ implies its approximation within $\rho=2-(1-\epsilon)=1+\epsilon, 0 \leqslant \epsilon \leqslant 1$. Then, the inapproximability bound $(743 / 742-\epsilon)$ of [7] for min_TSP12 together with theorem 1 conclude the proof.

\section{An improvement of the standard ratio for the maximum traveling salesman with distances 1 and 2}

We propose in this section a non-trivial improvement of the standard-approximation ratio for max_TSP12, by proving the following theorem. 


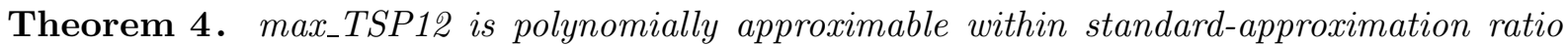
bounded below by $7 / 8$.

Proof. Combining, as in the proof of proposition 4 , expressions $\delta_{\max \_T S P 12} \geqslant 3 / 4, \omega_{\max }\left(K_{n}\right) \geqslant$ $a n$ and $\beta_{\max }\left(K_{n}\right) \leqslant b n$, one deduces $\rho_{\max \text { _TSP12 }} \geqslant(3 / 4)+(a / 4 b)$. Setting $a=1$ and $b=2$, the result claimed follows immediately.

Remark 2. Consider now the following simple way to use the algorithm of [11] in order to solve max_TSP12 on a graph $K_{n}$ with edge-distances 1 and 2 . The graph $\bar{K}_{n}$ in the first line of the algorithm MTSPALG just above is such that the distance of an edge $e$ of $\bar{K}_{n}$ is 1 if $e$ has distance 2 in $K_{n}$, and 2 if $e$ has distance 1 in $K_{n}$.

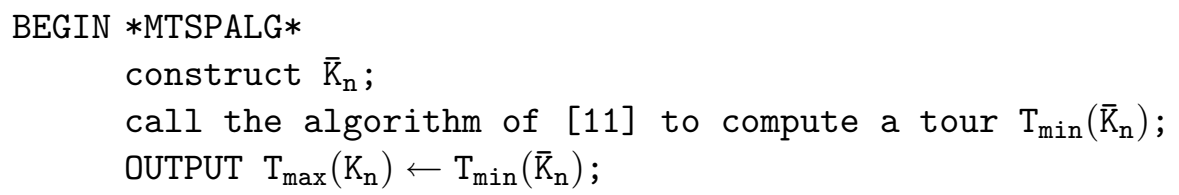

END.

Let us denote by A the $7 / 6$-algorithm of [11] called in the second line of MTSPALG. Then, by theorem $1, \beta\left(K_{n}\right)=3 n-\beta\left(\bar{K}_{n}\right)$ and $\lambda_{\text {MTSPALG }}\left(K_{n}\right)=3 n-\lambda_{\mathrm{A}}\left(\bar{K}_{n}\right)$, where $\beta\left(K_{n}\right)$ is the optimal value for max_TSP12 on $K_{n}$ and $\beta\left(\bar{K}_{n}\right)$ is the optimal value for min_TSP12 on $\bar{K}_{n}$. Then, $\lambda_{\mathrm{A}}\left(\bar{K}_{n}\right) / \beta\left(\bar{K}_{n}\right) \leqslant 7 / 6$, together with $\beta\left(\bar{K}_{n}\right) \geqslant n$ imply $\lambda_{\text {MTSPALG }}\left(K_{n}\right) / \beta\left(K_{n}\right) \geqslant 2 / 3$.

Note finally that standard-approximation ratio $7 / 8$ can be obtained by the following direct method.

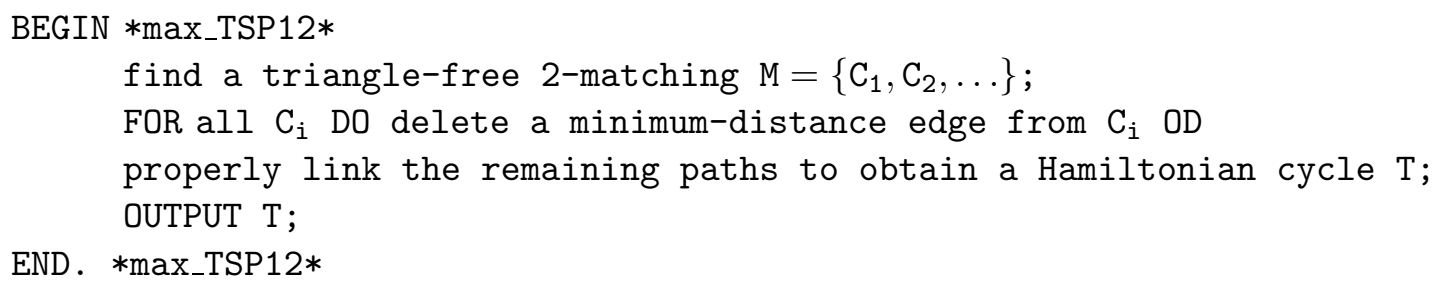

Let $p$ be the number of cycles of $M$ where 2-edges have been removed during the FoR-loop of algorithm max_TSP12. Then, $\lambda_{\max \_ \text {TSP12 }}\left(K_{n}\right) \geqslant d(M)-p, \beta\left(K_{n}\right) \leqslant d(M)$, and since $M$ is triangle-free, $d(M) \geqslant 8 p$. Consequently, $\lambda_{\max \_ \text {TSP12 }}\left(K_{n}\right) / \beta_{\max }\left(K_{n}\right) \geqslant 7 / 8$.

The above result, can be extended to the case of max_TSPab if we consider that here $\lambda_{\max \_ \text {TSP12 }}\left(K_{n}\right) \geqslant d(M)-p(b-a), \beta\left(K_{n}\right) \leqslant d(M)$ and $d(M) \geqslant 4 b p$. Hence the following corollary holds and concludes the paper.

Corollary 2. max_TSPab is polynomially approximable within standard-approximation ratio bounded below by $(3 / 4)+(a /(4 b))$.

\section{References}

[1] A. Aiello, E. Burattini, M. Furnari, A. Massarotti, and F. Ventriglia. Computational complexity: the problem of approximation. In C. M. S. J. Bolyai, editor, Algebra, combinatorics, and logic in computer science, volume I, pages 51-62, New York, 1986. North-Holland.

[2] G. Ausiello, A. D'Atri, and M. Protasi. Structure preserving reductions among convex optimization problems. J. Comput. System Sci., 21:136-153, 1980. 
[3] G. Ausiello, A. Marchetti-Spaccamela, and M. Protasi. Towards a unified approach for the classification of NP-complete optimization problems. Theoret. Comput. Sci., 12:83-96, 1980 .

[4] M. Bellare and P. Rogaway. The complexity of approximating a nonlinear program. Math. Programming, 69:429-441, 1995.

[5] M. Demange, P. Grisoni, and V. T. Paschos. Differential approximation algorithms for some combinatorial optimization problems. Theoret. Comput. Sci., 209:107-122, 1998.

[6] M. Demange and V. T. Paschos. On an approximation measure founded on the links between optimization and polynomial approximation theory. Theoret. Comput. Sci., 158:117-141, 1996.

[7] L. Engebretsen and M. Karpinski. Approximation hardness of TSP with bounded metrics. Report 89, Electr. Colloq. Computational Comp., 2000.

[8] M. R. Garey and D. S. Johnson. Computers and intractability. A guide to the theory of NP-completeness. W. H. Freeman, San Francisco, 1979.

[9] D. B. Hartvigsen. Extensions of matching theory. PhD thesis, Carnegie-Mellon University, 1984.

[10] J. Monnot, V. T. Paschos, and S. Toulouse. Differential approximation results for the traveling salesman problem. Cahier du LAMSADE 172, LAMSADE, Universit Paris-Dauphine, 2000 .

[11] C. H. Papadimitriou and M. Yannakakis. The traveling salesman problem with distances one and two. Math. Oper. Res., 18:1-11, 1993.

[12] A. I. Serdyukov. An algorithm with an estimate for the traveling salesman problem of the maximum. Upravlyaemye Sistemy, 25:80-86, 1984.

[13] S. A. Vavasis. Approximation algorithms for indefinite quadratic programming. Math. Programming, 57:279-311, 1992.

[14] E. Zemel. Measuring the quality of approximate solutions to zero-one programming problems. Math. Oper. Res., 6:319-332, 1981. 